\title{
Auditoría de oxígenoterapia de pacientes hospitalizados en establecimientos del Servicio de Salud Talcahuano
}

\author{
PATRICIO RIOSECO S.*, NICOLÁS RODRÍGUEZ O.**, STEPHAN SKOG M.**, \\ EDUARDO ROZAS C.** y ALDO SEPÚLVEDA N.**
}

\begin{abstract}
Audit on oxygen therapy in hospitalized patients from Public Health Service of Talcahuano, Chile
\end{abstract}

Oxygen is a commonly used drug in clinics and its use must be judicious. There are guidelines for oxygen therapy but we ignore if these are respected in our country. We conducted an audit of oxygen therapy by applying a survey to 381 patients in the three hospitals of Talcahuano Public Health Service. The day of the audit 13.7\% of the hospitalized patients were on oxygen, most of them with respiratory $(46.15 \%)$ or cardiovascular (25\%) diseases. Indication of $\mathrm{O}_{2}$ administration was given by a physician in $88.5 \%$ and there was not registry in $3.8 \%$ of the cases. There was not foundation for supplying $\mathrm{O}_{2}$ in $13.3 \%$ of patients. A fixed dose was indicated in $75 \%$ of cases and $50 \%$ had an oximetry value as a target. Oxygen was administered in most of the cases by nasal prongs and Venturi masks. Monitoring was based on pulse oximetry in the less complex hospitals and on arterial blood gases in the tertiary hospital. $100 \%$ of patients at urgency ward were receiving a different dose from that indicated at their admission time and none of them had a registry of the new dose. Mean duration of therapy was 7.8 days. We believe our results might represent what is going on with oxygen therapy in our country; having a good foundation and monitoring, we still don t use targets and there is a bad system of registry. We think that it would be advisably to carry out audits on oxygen therapy at national level on regular basis.

Key words: Oxygen; oxygen inhalation therapy; registries; survey and questionnaires.

\section{Resumen}

El oxígeno $\left(\mathrm{O}_{2}\right)$ es una de las drogas más utilizadas en clínica, su uso no está exento de riesgos. Existen guías internacionales para su uso, pero en nuestro medio no sabemos si se aplican. Nuestro objetivo fue describir la forma en que se utiliza y controla la oxígenoterapia en los 3 hospitales de la red del Servicio de Salud Talcahuano. Aplicamos una encuesta un día de agosto de 2016 a los pacientes hospitalizados en las instituciones de la red que recibian $\mathrm{O}_{2}$ en ese momento. Se recolectaron datos sobre la prescripción, administración y seguimiento de la terapia de $\mathrm{O}_{2}$. De los 381 pacientes auditados, un 13,7\% recibía oxígenoterapia. Los diagnósticos más frecuentes fueron de causa respiratoria (46,15\%) y cardiológica (25\%). La indicación la dio un médico en $88,5 \%$ de los casos y en un 3,8\% no había registro. En un 17,3\% de los pacientes no había fundamento para la indicación. Se indicó una dosis fija en el 75\% con una meta de $\mathrm{SaO}_{2}$ en el 50\%, siendo naricera y máscara de Venturi los métodos de administración más frecuentes monitorizándose con oximetría de pulso en los hospitales menos complejos y gasometría arterial en el hospital terciario. La duración media de la oxígenoterapia fue de 7,8 dias. Habiendo un buen fundamento y control de la oxígenoterapia aún no se indican metas a obtener. No hay un buen registro de la indicación ni de los cambios realizados. Creemos útil la realización periódica de este tipo de control para optimizar su uso evitando los potenciales efectos adversos en los pacientes.

Palabras clave: Oxígeno; oxígenoterapia; registros; encuesta y cuestionarios.

* Unidad de Enfermedades Respiratorias Hospital Las Higueras, Talcahuano, Chile.

** Becados de la Especialidad de Medicina Interna, Universidad de Concepción, Chile. 


\section{Introducción}

\section{Origen del oxígeno en la biósfera}

Nuestro universo y con él, nuestro planeta nació hace más o menos 4,5 billones de años y las primeras manifestaciones de vida, se encuentran hace 3,5 billones de años atrás en la forma de protobacterias y arqueas, organismos muy simples, unicelulares y anaeróbicos. En ese momento, la atmósfera terrestre prácticamente no tenía oxígeno $\left(\mathrm{O}_{2}\right)$ en su composición (se estima que tenía tan sólo $0,001 \%$ ).

La actividad fotosintética de estas protobacterias, determinó el primer evento de oxidación en la biósfera. Estas bacterias, obtenían su energía de la luz solar y eliminaban $\mathrm{O}_{2}$; como consecuencia hace alrededor de 2 billones de años, la concentración de $\mathrm{O}_{2}$ atmosférico aumentó significativamente dando origen a las células eucarióticas, que son las células constitutivas de todos los organismos mayores que una bacteria. Según la teoría endosimbiótica aún vigente, las protobacterias que producían $\mathrm{O}_{2}$, fueron progresivamente "engullidas" por las células eucarióticas y las mitocondrias de nuestras células serían el remanente de esas células iníciales. Sabemos que es en las mitocondrias donde ocurre la respiración celular aeróbica cuyo producto final es la producción de energía en forma de ATP. Existe evidencia paleontológica que en relación a este primer evento de oxidación, la vida se complejizó en la tierra. Hace 1 billón de años, otro evento de oxidación dio origen a los animales $y$, cuando la concentración atmosférica de $\mathrm{O}_{2}$ llegó alrededor de un $21 \%$, recién apareció la vida humana. Por lo tanto, el $\mathrm{O}_{2}$ no es necesario para que exista vida, pero nosotros nos debemos al $\mathrm{O}_{2} \mathrm{y}$ este, es a su vez originado por la actividad vital ${ }^{1}$. Cualquier aumento en la concentración de $\mathrm{O}_{2}$ que respiremos es anormal y por lo tanto, puede ser deletéreo ${ }^{2}$.

\section{Descubrimiento del oxígeno}

Entre 1772 y 1775 el químico inglés Joseph Priestley estaba dedicado a buscar nuevos gases. Al calentar monóxido de mercurio obtuvo dos vapores: uno se condensó en gotas, el mercurio, y el otro no se condensó. Priestley juntó ese gas en un recipiente e hizo algunos ensayos: si introducía una vela, esta ardía en forma más intensa; si acercaba ratones vivos, éstos se volvían muy activos. En vista de lo cual, él mismo inhaló un poco de ese gas a través de un embudo y notó que se sentía muy "ligero y cómodo". A este gas lo llamó "aire desflogistizado", hoy sabemos que era oxígeno. Sin saberlo, Priestley fue la primera persona o el primer paciente que usó una mascarilla de oxígeno. Sin embargo, también observó que si sus animales permanecían largo tiempo en contacto con este aire tan puro, su vitalidad se agotaba y morían es decir, algo de dañino tenía el nuevo gas descubierto ${ }^{3}$.

Generalmente se acepta que Priestley fue el descubridor del $\mathrm{O}_{2}$. Sin embargo, unos tres años antes que él, en Suecia otro experimentador obtuvo resultados similares a los de Priestley, pero como no era muy conocido en esa época, los editores científicos desestimaron sus resultados y no publicaron sus trabajos. Se trataba de Carl Wilhelm Scheele, un químico-farmacéutico sueco-alemán que en 1773 ya había descubierto lo que llamó "el gas del fuego", por su efecto de aumentar la combustión ${ }^{4}$. El trascendental descubrimiento del $\mathrm{O}_{2}$, entonces es compartido entre estos dos investigadores.

\section{Uso clínico del oxígeno}

En clínica, el $\mathrm{O}_{2}$ es probablemente una de las drogas más utilizadas, pero no existe real conciencia entre quienes la indican que su uso debe ser extremadamente cuidadoso ya que como cualquier droga, su uso correcto indudablemente salva vidas, pero es frecuente que también produzca daño ${ }^{5,6}$. Un grupo de pacientes especialmente lábil a los efectos adversos del $\mathrm{O}_{2}$ suplementario, son los portadores de Enfermedad Pulmonar Obstructiva Crónica (EPOC), que ingresan a un servicio de urgencia por una exacerbación aguda de su condición. No es infrecuente ver llegar a las salas de emergencia a un paciente que en su domicilio, respiraba en forma espontánea, y que al ingreso debe ser rápidamente conectado a ventilación mecánica para salvarle la vida. En tal condición existe en el pulmón un deterioro aún mayor de la desigualdad de la relación ventilación/ perfusión (V/Q) que la que basalmente tienen y se está empleando al máximo el mecanismo de vasoconstricción hipóxica pulmonar que impide la perfusión de zonas pobremente ventiladas. El uso de altas concentraciones de $\mathrm{O}_{2}$, como ocurre habitualmente en los traslados de estos enfermos hacia un centro asistencial, al aumentar la $\mathrm{PO}_{2}$ alveolar $\left(\mathrm{PAO}_{2}\right)$ disminuye la mencionada vasoconstricción hipóxica y su consecuencia es un empeoramiento de la relación V/Q que produce un aumento de la $\mathrm{PCO}_{2}$ en la sangre arterial ${ }^{7,8}$. Por otra parte, a medida que la $\mathrm{PO}_{2}$ en la sangre arterial aumenta gracias a la oxígenoterapia empleada, aumenta la hemoglobina oxigenada y esta tiene menor capacidad de unión al $\mathrm{CO}_{2}$ lo que lleva a una desviación hacia la derecha en la curva de disociación de $\mathrm{CO}_{2}$ de la hemoglobina que 
resulta también en un aumento en la retención de $\mathrm{CO}_{2}{ }^{9}$. Unidos estos mecanismos, pueden llevar al enfermo a una narcosis por $\mathrm{CO}_{2}^{7,10}$.

El oxígeno es una droga y como tal, debe ser indicado en una dosis definida, con un objetivo claro, mediante un sistema de administración identificado y monitorizado pues tanto la hipoxemia como la hiperoxemia son dañinas para el paciente ${ }^{11}$. De hecho, se ha comunicado una mortalidad en exceso cuando se usan dosis elevadas de $\mathrm{O}_{2}$ en pacientes con EPOC en la etapa pre hospitalaria ${ }^{12}$. Es común ver en nuestras salas de hospitalización de adultos, pacientes a los cuales se aumenta la $\mathrm{FiO}_{2}$ durante la noche, por diferentes causas (aumento de la disnea de base, ansiedad, polipnea), que despiertan en la mañana con una aceptable $\mathrm{PaO}_{2}$ pero con una mayor retención de $\mathrm{CO}_{2}$. Esto ha sido demostrado en un elegante y práctico trabajo de Samolski y cols., quienes concluyeron que el aumento de la $\mathrm{FiO}_{2}$ nocturna en pacientes EPOC con hipercapnia diurna estable causaba una peligrosa mayor acidosis respiratoria a pesar de mejorar la oxigenación nocturna ${ }^{13}$.

\section{Cumplimiento de las guías de oxígenoterapia}

Debemos entender que el papel de la oxígenoterapia es uno solo: mejorar la hipoxemia.

Existen guías y excelentes revisiones críticas para su utilización en diferentes patologías, pero en nuestro país y en particular en nuestro medio no sabemos si éstas se respetan ${ }^{5,14,15}$. En 2016, Cousins y cols., revisaron los estudios publicados desde 1980 a 2015 acerca de la oxígenoterapia y encontraron 48 comunicaciones acerca del uso de esta droga, las que se refieren especialmente a guías y su cumplimiento, siendo la mayoría provenientes del Reino Unido ya que allí, desde el año 2008 en adelante, se hacen auditorías anuales. Le siguen en frecuencia de publicaciones Nueva Zelandia y Canadá ${ }^{16}$. En Chile no hay publicaciones y no existe ningún control periódico de este tipo por lo que hemos considerado de importancia tanto desde el punto de vista clínico como del punto de vista operacional y de costos, indagar sobre cómo se usa el oxígeno en el Servicio de Salud de Talcahuano (SSThno), en el que trabajamos.

El propósito de este estudio es describir la forma en que se utiliza y controla la oxígenoterapia en pacientes hospitalizados en establecimientos del Servicio de Salud Talcahuano que cuenta con un hospital de alta complejidad: hospital Las Higueras, uno de mediana complejidad: hospital de Tomé y un hospital básico: hospital de Lirquén, lo cual podría ser una muestra representativa de la realidad nacional.

\section{Material y Métodos}

Se solicitó a los jefes de Servicios Clínicos de adultos del hospital terciario y a los directores de los hospitales, básico y de mediana complejidad la autorización para realizar la auditoría un día del mes de agosto de 2016 en un momento determinado de la mañana.

La actividad fue autorizada por la Unidad de Calidad y Seguridad de la Atención y la Unidad de Regulación de la Investigación de nuestro establecimiento y puesto que se trataba sólo de una revisión de datos de una acción habitualmente realizada en nuestros establecimientos y no un estudio experimental, no se consideró necesario someter a los pacientes o sus representantes a un proceso de consentimiento informado.

Se confeccionó una encuesta preguntando datos demográficos, tipo de profesional que indicó el oxígeno, diagnóstico de base y fundamento de la indicación (gasometría arterial y/u oximetría de pulso), método de administración, objetivo a alcanzar, medio de monitorización, cambios efectuados y duración de la oxígenoterapia hasta el día de la auditoría. En todo momento se resguardó la confidencialidad de los datos personales de cada paciente.

Tres de los autores (NR, SK y ER) aplicaron la encuesta en cada uno de los tres hospitales del Servicio de Salud Talcahuano, utilizando las fichas clínicas y tablas de indicaciones de los pacientes adultos hospitalizados que ese día estaban recibiendo oxígeno. Existe el antecedente de un intento previo que realizamos en el año 2013 en que solicitamos a cada director y jefe de servicio designar a un miembro de su personal para aplicar una encuesta similar, pero el estudio fue frustrado por la inexactitud de los registros obtenidos, la gran variabilidad en el momento de la aplicación de la encuesta y la falta de datos completos en más de la mitad de los encuestados, muy probablemente por no ser personal clínico quien estuvo a cargo del proceso.

Los datos fueron analizados de acuerdo a porcentaje de cumplimiento de los puntos de la encuesta.

\section{Resultados}

De 381 pacientes adultos hospitalizados en el Servicio de Salud Talcahuano, el día de la auditoría el 13,7\% estaba recibiendo algún tipo de oxígenoterapia; ( $10 \%$ en el hospital básico, $11 \%$ en el hospital de mediana complejidad y $13,7 \%$ en el hospital de alta complejidad) 55,7\% eran mujeres 
y $44,3 \%$ varones, con un promedio de edad de 62,2 años y un rango entre 20 y 100 años de edad. La mayor parte de los diagnósticos $(46,15 \%)$ eran respiratorios, $25 \%$ cardiológico, luego una miscelánea de diagnósticos con un $17,3 \%$, un $5,77 \%$ infecciosos (sepsis) y un 5,77\% de causas neurológica (Figura 1). En el hospital terciario, como es de suponer la UCI era el servicio que más pacientes tenía en oxígenoterapia $(83,3 \%)$, seguido por Medicina Interna (18,98\%), luego el Servicio de Urgencia (12,9\%) y con un mínimo los servicios médico quirúrgicos (urología, traumatología, otorrinolaringología y oftalmología) $(2,79 \%)$ (Figura 2).

En el Servicio de Salud Talcahuano en su globalidad, la indicación del $\mathrm{O}_{2}$ la dio un médico en el $88,5 \%$ de los casos; una enfermera en el $7,7 \%$ de los casos y en un 3,8\% no había registro del profesional responsable de la indicación. En el $100 \%$ de los casos en los hospitales menos complejos, la indicación fue dada por un médico mientras que en el hospital de mayor compleji-

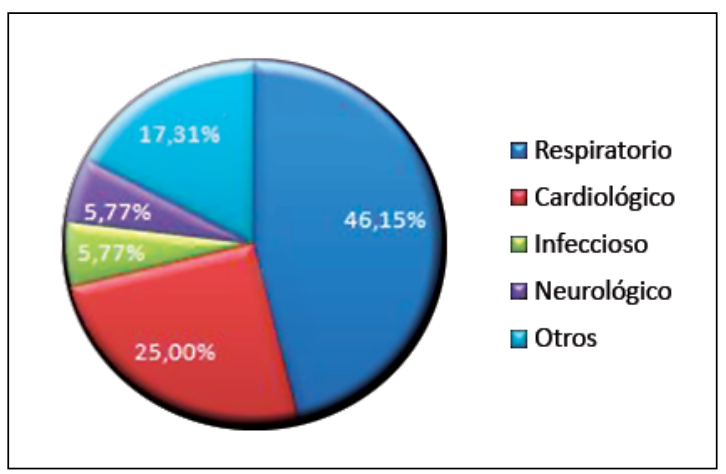

Figura 1. Diagnóstico principal de los pacientes hospitalizados en oxígenoterapia en los 3 hospitales del Servicio de Salud Talcahuano. dad, un $75 \%$ de las indicaciones fue médica, un $5,77 \%$ por enfermera, un $1,9 \%$ por otro profesional (kinesiólogo) y lo que es importante, en un $17,3 \%$ no había registro de la indicación por un profesional responsable, es decir, no se sabía quién había indicado la oxígenoterapia (Figura 3).

En los hospitales básico y de mediana complejidad, la oxígenoterapia estaba fundamentada en el $100 \%$ de los casos mientras que en el hospital de alta complejidad, sólo el $65,4 \%$ tenía algún fundamento y había un $17,3 \%$ de los casos en que no había fundamentación y una cifra similar en que se había comenzado la oxígenoterapia por paro cardiorrespiratorio no habiendo gasometría arterial o $\mathrm{SaO}_{2}$ de base. En general en el SSThno, la oxígenoterapia se fundamentaba bien en el $80,77 \%$ de los casos, no habiendo fundamentos en el $17,3 \%$ y no había registro en el 1,9\% de los pacientes (Figura 4).

En el hospital básico se indicaba una dosis fija en el $75 \%$ y sólo en el $50 \%$ de las indicaciones se esperaba un objetivo determinado. En el hospital

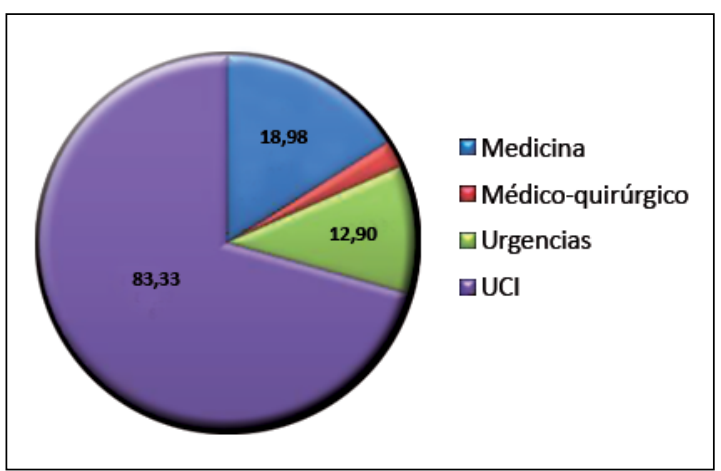

Figura 2. Distribución porcentual por servicio clínico de los pacientes en oxígenoterapia en Hospital Las Higueras, Servicio de Salud Talcahuano.

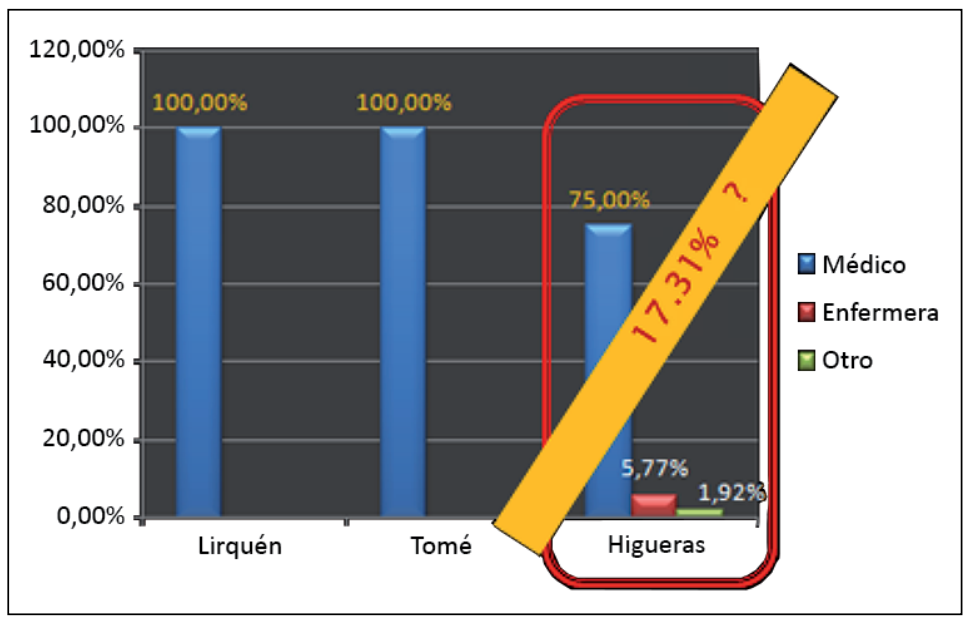

Figura 3. Profesional que indicó oxígenoterapia en los hospitales del Servicio de Salud Talcahuano. Lirquén; hospital de Lirquén; Tomé: hospital de Tomé: Higueras: hospital Las Higueras, Talcahuano. En este hospital no existía registro del profesional que indicó oxígenoterapia en $17,3 \%$ de los pacientes que recibió $\mathrm{O}_{2}$ suplementario. 
Fundamentos gases art $/ \mathrm{SaO}_{2}$ SSTHNO

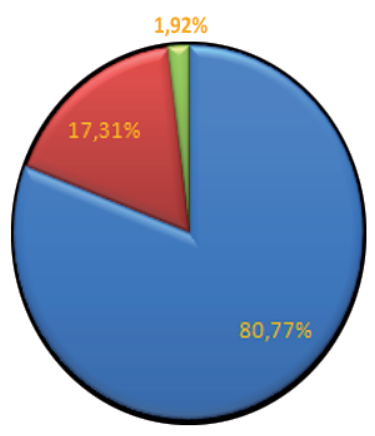

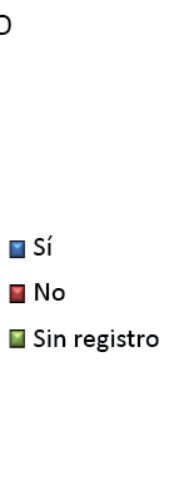

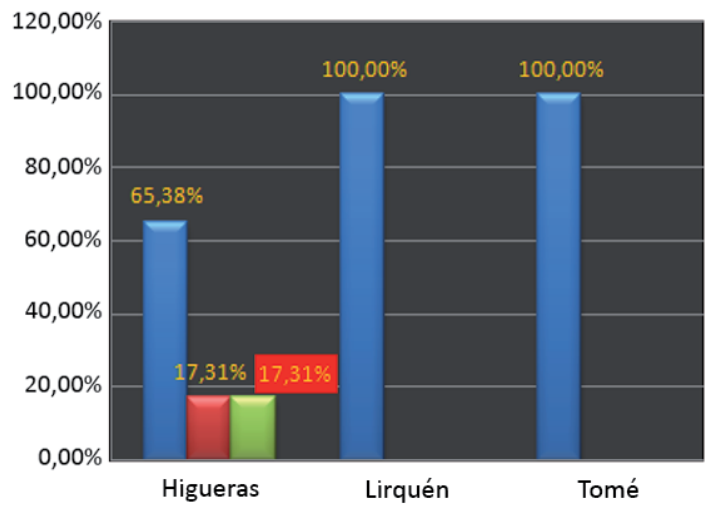

Figura 4. Fundamentación de la oxígenoterapia en el Servicio de Salud Talcahuano (SSTHNO) y en sus tres hospitales: Las Higueras (Higueras), Lirquén y Tomé. Gases art.: gasometría arterial. $\mathrm{SaO}_{2}$ : saturación de oxihemoglobina evaluada por oximetría de pulso.

de mediana complejidad sólo en el $50 \%$ de los pacientes se indicaba una dosis fija y en el $75 \%$ de los casos se esperaba un objetivo o meta a obtener (Tabla 1).

En el hospital de alta complejidad en el Servicio de Medicina se indicó una dosis fija en el $73 \%$ y sólo en el $46,67 \%$ una meta a obtener. No había registro de la meta a obtener en $6,66 \%$ de los casos. En los servicios quirúrgicos (otros) en la mitad de los casos se indicó una dosis determinada y en el 50\% una meta. En el servicio de Urgencias se indicó una dosis fija en la mitad de los casos con un objetivo también en la mitad. Llama la atención que en la Unidad de Pacientes
Críticos (UPC) se indicaba una dosis de $\mathrm{O}_{2}$ en más de la mitad de los casos $(66,7 \%)$ y en menos de un tercio $(23,8 \%)$ se esperaba una meta definida (Tabla 2).

En la Figura 5 se muestra las diferentes formas de administración de $\mathrm{O}_{2}$ en los hospitales del SSThno y se destaca esta información para el hospital de mayor complejidad en cada uno de sus servicios, así podemos ver que la naricera fue el método más utilizado en los hospitales básico y de mediana complejidad así como también en los Servicios de Medicina y de menor complejidad del hospital terciario. En la UPC de este último, como es esperable, una importante proporción de

Tabla 1. Tipo de indicación de la oxígenoterapia: dosis fija y meta a obtener en los tres hospitales del Servicio de Salud Talcahuano

\begin{tabular}{|lcccccc|}
\hline SSTalcahuano & & Dosis fija (\%) & & \multicolumn{3}{c|}{ Meta a obtener (\%) } \\
Hospitales & Sí & No & Sin registro & Sí & No & Sin registro \\
\hline Lirquén & 75,00 & 25,00 & 0,00 & 50,00 & 50,00 & 0,00 \\
Tomé & 50,00 & 50,00 & 0,00 & 75,00 & 25,00 & 0,00 \\
\hline Las Higueras & 55,77 & 11,54 & 32,69 & 36,36 & 61,63 & 2,01 \\
\hline
\end{tabular}

Tabla 2. Tipo de indicación de la oxígenoterapia: dosis fija y meta a obtener en los servicios clínicos del hospital Las Higueras de Talcahuano

\begin{tabular}{|c|c|c|c|c|c|c|}
\hline \multirow{2}{*}{$\begin{array}{l}\text { Hospital Las Higueras } \\
\text { Servicios clínicos }\end{array}$} & \multicolumn{3}{|c|}{ Dosis fija (\%) } & \multicolumn{3}{|c|}{ Meta a obtener (\%) } \\
\hline & Sí & No & Sin registro & Sí & No & Sin registro \\
\hline Medicina & 73,33 & 13,33 & 13,34 & 46,67 & 46,67 & 6,66 \\
\hline Urgencias & 50,00 & 50,00 & 0,00 & 50,00 & 50,00 & 0,00 \\
\hline UPC & 66,67 & 9,52 & 23,81 & 23,81 & 76,19 & 0,00 \\
\hline Otros & 50,00 & 0,00 & 50,00 & 50,00 & 50,00 & 0,00 \\
\hline
\end{tabular}




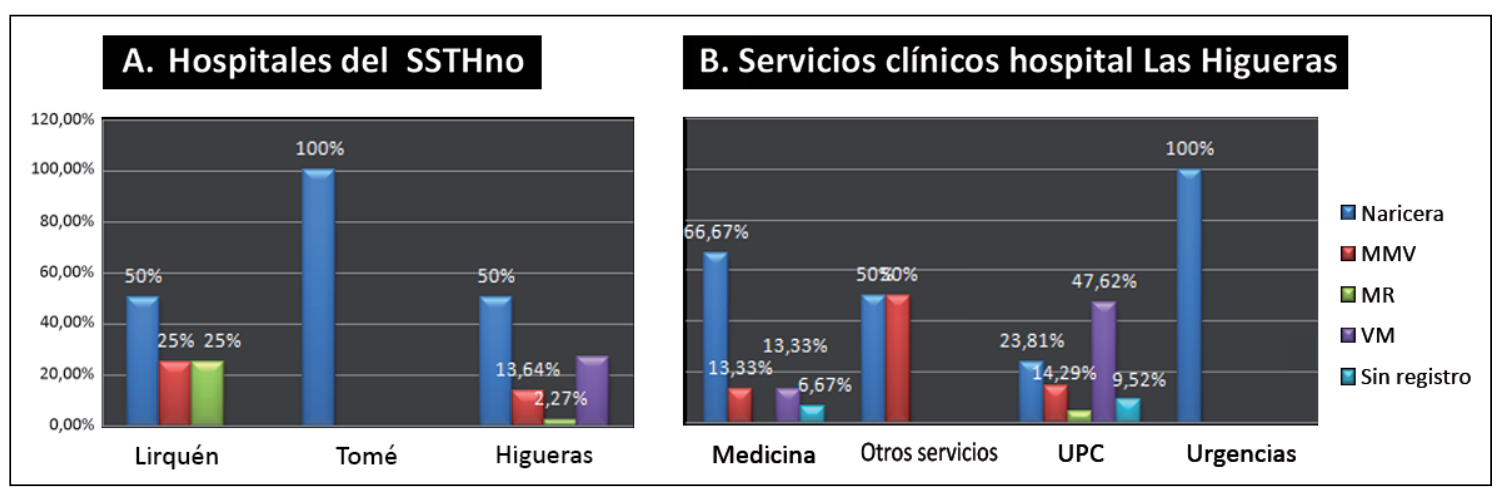

Figura 5. A: Método de administración de $\mathrm{O}_{2}$ en los 3 hospitales del Servicio de Salud Talcahuano: Lirquén, Tomé y Las Higueras. B: Método de administración de $\mathrm{O}_{2}$ en los servicios clínicos del hospital Las Higueras. UPC: unidad de pacientes críticos. MMV: máscara de Venturi; MR: máscara con reservorio; VM: ventilación mecánica.

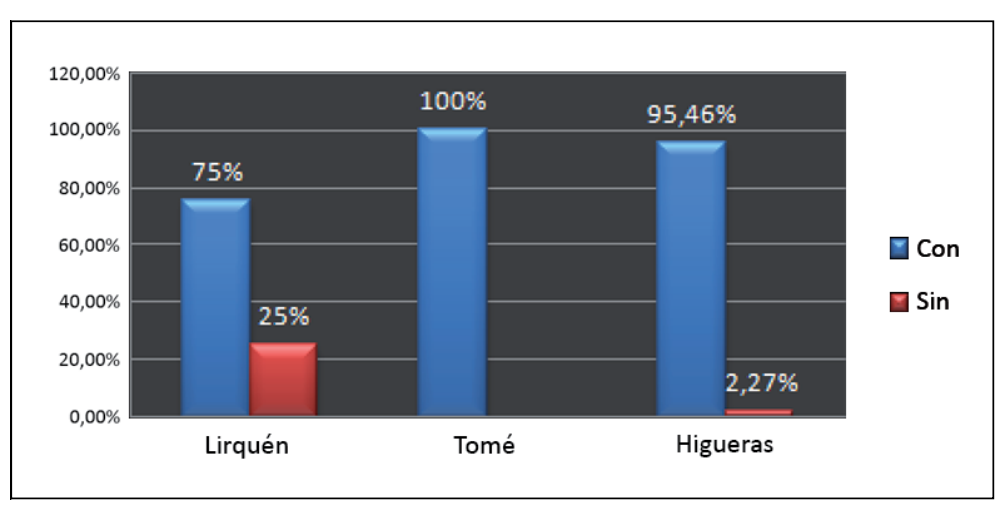

Figura 6. Monitorización de la oxígenoterapia en los hospitales de Lirquén, de Tomé y Las Higueras del Servicio de Salud Talcahuano. Se controló la administración de $\mathrm{O}_{2}$ con oximetría de pulso en los hospitales de Lirquén y Tomé y con gasometría arterial en el hospital Las Higueras. las oxígenoterapias eran administradas a través de ventilación mecánica (47,6\%). Es importante destacar que en casi un $10 \%$ de los casos en esta unidad no había registro del método de oxígenoterapia que estaba recibiendo el paciente.

Se controlaba la administración de $\mathrm{O}_{2}$ con oximetría de pulso en el $75 \%$ de los casos en el hospital básico y en el $100 \%$ de los casos en el hospital de mediana complejidad mientras que se controló el tratamiento con gasometría arterial en el 90 a $100 \%$ de los casos en el hospital terciario (Figura 6). No se observó diferencias entre los distintos servicios de este último establecimiento primando el control con gasometría arterial sobre la oximetría en la UPC.

Hubo cambios en la indicación de $\mathrm{O}_{2}$ desde la prescripción inicial hasta el día de la auditoría en el $75 \%$ de los casos en los hospitales básico y mediano, indicada por médico en el $100 \%$ de los casos en el hospital mediano y en el 33,33\% en el hospital básico desconociéndose en el resto el tipo de profesional que indicó el cambio. Dichos cambios se referían a la dosis de $\mathrm{O}_{2}$ usada y al método de administración, cambiándose en la mayoría de los casos de naricera a máscara de Venturi (100\% en hospital de Tomé y 66,7\% en hospital de Lirquén). En el hospital terciario hubo cambios en la dosis de $\mathrm{O}_{2}$ y en el método de administración en el 50\% de los casos médicoquirúrgicos, la mayoría indicados por médico y en el 70,6\% de los casos en UPC, pero aquí sólo el $58,8 \%$ de los cambios fue indicado por un médico, el 29,4\% por kinesiólogo y el resto, por enfermera. Es importante señalar que en el Servicio de Urgencias del hospital terciario, en el 100\% de los casos los pacientes estaban recibiendo una dosis diferente a la inicialmente indicada y este cambio no estaba registrado en la ficha clínica ni constaba en ella el profesional responsable de la indicación de cambio. En cuanto a cambios en la modalidad de oxígenoterapia, en el $17,65 \%$ de los pacientes de la UPC se escaló a ventilación mecánica y se desescaló a máscara de Venturi en el $23,5 \%$ de los casos.

La duración media del tratamiento de oxígenoterapia en el Servicio de Salud fue de 7,8 días en promedio por paciente con una variación de 1 a 40 días. 


\section{Discusión}

Desde la publicación de las Guías de Oxígenoterapia de la Sociedad Británica del Tórax en 2008, en el Reino Unido se hace una auditoría anual y se ha observado una mejoría substancial en el uso del $\mathrm{O}_{2}$ en los hospitales británicos gracias a campañas emprendidas de acuerdo a estos resultados, que pretenden subsanar las falencias observadas año a año. A pesar de dicha mejoría, persisten errores en las prescripciones especialmente en relación al registro de la prescripción y las metas en cuanto a $\mathrm{SaO}_{2}$ que se intenta obtener, hecho que redunda en un mayor riesgo de mortalidad para los enfermos bajo tratamiento ${ }^{10}$.

Si comparamos nuestros resultados con aquellos obtenidos en la última auditoría realizada en el Reino Unido en $2015^{10}$, vemos que un número similar de pacientes en un momento dado se encuentra recibiendo $\mathrm{O}_{2}$ suplementario en nuestro SSThno: $13,7 \%$ en 3 hospitales contra un $14 \%$ en 180 hospitales. En nuestro hospital terciario, con obligaciones docentes, había una cifra inaceptable de uso de $\mathrm{O}_{2}$ sin prescripción: $17,3 \%$ contra un estándar esperado de un $100 \%$ o un $93,4 \%$ observado por Neves y cols en Portugal ${ }^{9}$. La calidad de la prescripción es deficiente ya que sólo un $36,3 \%$ de los pacientes recibían una indicación de $\mathrm{O}_{2}$ con una meta definida (\% de saturación de oxihemoglobina a obtener), comparado con el $52,7 \%$ en el Reino Unido y con Portugal, en donde en sólo el 16,4\% de los pacientes en 24 hospitales reciben una prescripción con una meta de $\mathrm{SaO}_{2}$ a obtener 9 .

En general, se ha observado que la hiperoxemia prevalece en los pacientes de Unidades Críticas, especialmente en aquellos ventilados mecánicamente. La hiperoxia causa daño alveolar y aumenta la respuesta inflamatoria sistémica. Este hecho es a menudo ignorado y no se le da la importancia que tiene. Las cifras que observamos, en cuanto a la indicación y objetivos a obtener, en nuestra UPC así lo demuestran. Entre los pacientes críticamente enfermos, en nuestro hospital terciario, al $66,6 \%$ se le indicaba una dosis determinada de $\mathrm{O}_{2}$ y se esperaba una $\mathrm{SaO}_{2}$ definida sólo en un $23,8 \%$. Es por lo tanto muy probable que muchos de ellos, hayan sido expuestos a dosis inadecuadas de $\mathrm{O}_{2}$ y por lo tanto, a un riesgo de eventos adversos mayores no deseados.

A este respecto creemos interesante destacar la publicación de Girardis y cols., que demuestra que entre 434 pacientes críticos tratados en UCI con oxígenoterapia, aquellos sometidos a la terapia convencional en este tipo de unidades es decir, pacientes que mantienen una $\mathrm{SaO}_{2}$ de 97 a 100\% tenían una tasa de mortalidad mucho mayor que aquellos que recibían una oxígenoterapia más conservadora es decir, manteniendo una $\mathrm{SaO}_{2}$ de 94 a $98 \%$ (mortalidad 20,2 versus $11,6 \%)^{17,18}$.

Una deficiencia de nuestro método fue no investigar, con respecto a los cambios en la dosis y/o en el método de administración de $\mathrm{O}_{2}$, su eventual relación con la obtención de la $\mathrm{SaO}_{2}$ meta, en los casos en que esta fue definida y al mismo tiempo, si al momento de la auditoría, la $\mathrm{SaO}_{2}$ observada era la que se había indicado originalmente. No podemos por lo tanto evaluar si los cambios que se realizaron fueron adecuados a la condición del enfermo por un lado o si ellos respondieron a una indicación definida (ej.: si al obtener tal $\mathrm{SaO}_{2}$, se indicó disminuir o mantener la dosis en uso).

Nuestro estudio tiene limitaciones importantes por el número reducido de pacientes y hospitales auditado que puede no necesariamente, -contrario a lo que pensamos- reflejar la realidad nacional. Sin embargo, en nuestro conocimiento es la primera vez que se realiza en Chile una auditoría de este tipo y ella nos señala que no estamos siguiendo guías internacionalmente validadas en el uso medicinal del $\mathrm{O}_{2}$.

Nuestro estudio pone de manifiesto la necesidad de unificar las recomendaciones de la oxígenoterapia en nuestro medio, las cuales se encuentran diseminadas en publicaciones acerca de diversas patologías, con el fin de uniformar su uso.

\section{Conclusiones}

Podemos concluir que existiendo una buena fundamentación para el uso de $\mathrm{O}_{2}$ en la mayoría de los casos en nuestro Servicio de Salud de Talcahuano, existe un control aún no óptimo del mismo; con un desconocimiento importante de qué se desea obtener con dicho tratamiento (no hay metas) y un registro muy deficiente de los cambios efectuados, tanto en las dosis, medios de administración como del profesional responsable de la indicación de dicho cambio. Es muy posible entonces que haya algunos tratamientos con $\mathrm{O}_{2}$ que no están indicados en absoluto o que estando indicados, por falta de control o de registro adecuados, se mantengan dosis supra o sub-óptimas poniendo en riesgo a los pacientes.

Es probable que haya un insuficiente conocimiento por parte del conjunto de médicos, enfermeras y kinesiólogos en lo que se refiere tanto a los métodos de administración del $\mathrm{O}_{2}$, como una falta de comprensión de sus efectos, como tam- 
bién del papel y de los riesgos de la oxígenoterapia. Es también probable que las deficiencias en los registros estén dadas por la carga asistencial del personal como también por la incorporación de nuevos profesionales involucrados tanto en el control como en la monitorización de la oxígenoterapia.

Creemos pertinente recomendar una adecuada diseminación de guías de oxígenoterapia en los centros asistenciales de nuestro país, así como también una monitorización periódica de su correcta utilización.

\section{Bibliografía}

1.- MARGULIS L. Symbiosis in Cell Evolution: Life and its environment on the early earth. W.H. Freeman, San Francisco, Ca.XXII+419pp 1981.New York 1993.

2.- CLARK J M, LAMBERSTEN C J, GELFAND R, FLORES N D, PISARELLO J B, ROSSMAN M D et al. Effects of prolonged oxygen exposure at 1.5, 2.0, or 2.5 ATA on pulmonary function in men (Predictive Studies V). J Appl Physiol 1999; 86: 243-59.

3.- AMERICAN CHEMICAL SOCIETY INTERNATIONAL HISTORIC CHEMICAL LANDMARKS. Discovery of Oxygen by Joseph Priestley. Disponible en: https://www.acs.org/content/acs/en/education/whatischemistry/landmarks/josephpriestleyoxygen.html (Consultado el 18 de julio de 2017).

4.- CASTLE F A. "Carl Wilhelm Scheele" American Druggist Vol. 15 august. 1886.

5.- BLAKEMAN T C. Evidence for oxygen use in the hospitalized patient: is more really the enemy of good? Respir Care. 2013; 58: 1679-93.

6.- MARTIN D S, GROCOTT M P W. Oxygen therapy and anaesthesia: too much of a good thing? Anaesthesia 2015; 70: 518-22.

7.- BRILL S E, WEDZICHA J A. Oxygen therapy in acute exacerbations of chronic obstructive pulmonary disease. Int J Chron Obstruct Pulmon Dis 2014; 9: 1241-52.

8.- ROBINSON T D, FREIBERG D B, REGNIS J A, YOUNG I H. The role of hypoventilation and ventilation-perfusion redistribution in oxygen induced hyper- capnia during acute exacerbations of chronic obstructive pulmonary disease. Am J Respir Crit Care Med 2000; 16: $1524-9$.

9.- NEVES J T, LOBAO M J, EMO working group. Oxygen therapy multicentric study. A nation wide audit to oxygen therapy procedures in Internal Medicine wards. Rev Port Pneumol 2012; 18: 80-5.

10.- O'DRISCOLL B R. British Thoracic Society. Emergency Oxygen Audit Report. National Audit Period 15 August-1 November 2015.

11.- MARTIN D S, GROCOTT M P. III Oxygen therapy in anaesthesia: the yin and yang of $\mathrm{O}_{2}$. Br J Anaesth 2013; 111: 867-71.

12.- WIJESINGHE M, PERRINK, HART K, CLAY J, WEATHERALL M, BEASLY R. Pre-hospital oxygen therapy in acute exacerbations of chronic obstructive pulmonary disease. Intern Med J 2011; 41: 618-22.

13.- SAMOLSKI D, TÀRREGA J, ANTÒN A, MAYOS M, MARTÍ S, FARRERO E et al Sleep hypoventilation due to increased nocturnal oxygen flow in hypercapnic COPD patients. Respirology. 2010;15:283-8. Doi:10.1111/j.1440-1843.2009.01665.x.Epub 2009 Nov 23.

14.- MINISTERIO DE SALUD, GOBIERNO DE CHILE. Manual Operativo Programas de Salud Respiratoria. División de Atención Primaria, Unidad de Salud Respiratoria, 2015.

15.- O'DRISCOLL B R, HOWARD L S, DAVIDSON A G. BTS Guideline for emergency oxygen use in adult patients. Thorax 2008; 63 Suppl. 6: vi 1-68.

16.- COUSINS J L, WARK P A B, MCDONALD V M. Acute oxygen therapy: a review of prescribing and delivery practices. Int J Chron Obstruct Pulmon Dis 2016; 11:1067-75. doi:10.2147/COPD.

17.- GIRARDIS M, BUSANI S, DAMIANI E, DONATI L, MARUDI A, MORELLI A et al. Effect of Conservative vs Conventional Oxygen Therapy on Mortality Among Patients in an Intensive Care Unit: The Oxygen ICU Randomized Clinical Trial. JAMA 2016, 18; 316: 15839. doi:10.1001/jama.2016.11993.

18.- PANNU S R. Too Much Oxygen: Hyperoxia and Oxygen Management in Mechanically Ventilated Patients. Semin Respir Crit Care Med 2016; 37: 16-22. doi:10.1055/s-0035-1570359.Epub 2016 jan 28.

Correspondencia a:

Dr. Patricio Rioseco S.

Unidad de Enfermedades Respiratorias

Hospital Las Higueras, Talcahuano, Chile.

Email: parioseco@gmail.com 